\title{
The role of the interlayer state in the electronic structure of superconducting graphite intercalated compounds
}

\author{
GÁBOR CSÁNYI* , P. B. LITLEWOOD, ANDRIY H. NEVIDOMSKYY, CHRIS J. PICKARD AND B. D. SIMONS
}

Cavendish Laboratory, Madingley Road, Cambridge CB3 OHE, UK

*e-mail: gc121@cam.ac.uk

A lthough not an intrinsic superconductor, graphite exhibits superconductivity when intercalated with certain dopants ${ }^{1}$. Perhaps the most studied of these graphite-based superconductors are the alkali metal-graphite intercalation compounds (GICs) (ref. 2), of which the easiest to fabricate is $\mathrm{C}_{8} \mathrm{~K}$ (ref. 3), with a transition temperature $T_{\mathrm{c}} \cong 0.14 \mathrm{~K}$ (ref. 2). By increasing the alkali metal concentration (through high-pressure fabrication techniques), the transition temperature can be increased up to $5 \mathrm{~K}$ in $\mathrm{C}_{2} \mathrm{Na}$ (ref. 4). Superconductivity in $\mathrm{C}_{6} \mathrm{Yb}$ and $\mathrm{C}_{6} \mathrm{Ca}$ with $T_{\mathrm{c}} \cong 6.5$ and $11.5 \mathrm{~K}$, respectively, and at ambient conditions has been observed ${ }^{5}$. Here we explore the architecture of the states near the Fermi level and identify characteristics of the electronic band structure generic to GICs. In addition to the expected charge transfer from the intercalant atoms to the graphene sheets, resulting from the occupation of the $\pi$ bands, we find that in all of those-and only those-compounds that superconduct, an interlayer state, which is well-separated from the carbon sheets, also becomes occupied. We show that the energy of the interlayer band is controlled by a combination of its occupancy and the separation between the carbon layers.

To focus our discussion, we begin with an investigation of the newly discovered $\mathrm{C}_{6} \mathrm{Yb}$ system. Our calculations rely on density functional theory (DFT) techniques ${ }^{6}$ applied in the local density approximation (LDA $)^{7}$. As the $4 f$ shell of $\mathrm{Yb}$ is found to be filled (in agreement with experiment), the DFT-LDA can be applied with some confidence. Moreover, one may infer that the $4 f$ orbitals play no essential role in superconductivity. The atomic structure of $\mathrm{C}_{6} \mathrm{Yb}$, shown in Fig. 1a, involves a stacked arrangement of graphene sheets intercalated with a triangular lattice configuration of $\mathrm{Yb}$ atoms. The stacking of the graphene sheets is AAA, whereas the $\mathrm{Yb}$ atoms occupy interlayer sites above the centres of the hexagons of the graphene sheets in an $\mathrm{ABAB}$ stacking arrangement leading to a $P 63 / M M C$ crystallographic structure. We use the same geometry for $\mathrm{C}_{6} \mathrm{Ca}$.

Figure 1d shows the results of the band structure calculation of $\mathrm{C}_{6} \mathrm{Yb}$ centred on the Fermi energy. To resolve the qualitative structure of the levels and identify the bands intersecting the Fermi level, it is helpful to compare the dispersion with the corresponding 'empty' graphite-like system and the 'empty' Yb metal, obtained simply by removing, respectively, the $\mathrm{Yb}$ and $\mathrm{C}$ atoms from the $\mathrm{C}_{6} \mathrm{Yb}$ structure whilst holding the lattice constants fixed. In $\mathrm{C}_{6} \mathrm{Yb}$, the previously unoccupied graphite $\pi^{*}$ bands are seen to intersect the new Fermi level (owing to the enlarged interlayer distance, each is almost doubly degenerate). Of these, one remains almost unperturbed by the presence of $\mathrm{Yb}$, whereas the degeneracy of the other is lifted and one of the resulting bands hybridizes strongly with a new band that also crosses the Fermi level. The latter resembles both the free-electron-like state of expanded $\mathrm{Yb}$ metal (Fig. 1c) and the interlayer band intrinsic to pure graphite (Fig. 1e). As well as the $5 s$ and $5 p$ orbitals (which lie well outside the range of energies shown in Fig. 1d), the $4 f$ orbitals associated with the two $\mathrm{Yb}$ atoms in the unit cell form a set of localized almost nondispersive bands that appear at about $0.8 \mathrm{eV}$ below the Fermi level. Referring to Fig. 2, and the discussion in ref. 8 , we note that a similar phenomenology applies to the band structure of $\mathrm{C}_{6} \mathrm{Ca}$.

Although the presence of the interlayer band and its hybridization with the $\pi^{*}$ band helps to explain the diminished normal state resistance anisotropy observed in $\mathrm{C}_{6} \mathrm{Yb}$ over that of pure graphite ${ }^{5}$, could its occupation be significant for superconductivity? To address this question, it is instructive to draw comparison with other superconducting GICs. It is known from low electron-energy-loss spectroscopy measurements of the superconducting compound $\mathrm{C}_{8} \mathrm{~K}$ that, in common with the $\mathrm{C}_{6} \mathrm{Yb}$ system, the interlayer band lies below the Fermi energy ${ }^{9}$. However, a more discerning test of the significance of the interlayer state is presented by the Li intercalates. Although intercalation at ambient pressure leads to the $\mathrm{C}_{6} \mathrm{Li}$ structure, high-pressure techniques can be used to fabricate $\mathrm{C}_{3} \mathrm{Li}$ and $\mathrm{C}_{2} \mathrm{Li}$ compounds. Significantly, whereas the $\mathrm{C}_{3} \mathrm{Li}$ system (like its cousin $\mathrm{C}_{6} \mathrm{Li}$ ) remains nonsuperconducting, $\mathrm{C}_{2} \mathrm{Li}$ has a transition at $T_{\mathrm{c}} \simeq 1.9 \mathrm{~K}$ (ref. 10). Referring to Figs 2 and 3, one may note that, in common with the $\mathrm{C}_{6} \mathrm{Yb}$ and $\mathrm{C}_{6} \mathrm{Ca}$ system, the Li GICs also exhibit a free-electron-like interlayer band. The dispersive character of this band is reflected clearly in the Fermi surface (see Fig. 3a). However, significantly, increasing the $\mathrm{Li}$ ion concentration (and, with it, the degree of electron doping) leads to a lowering of the interlayer band resulting 

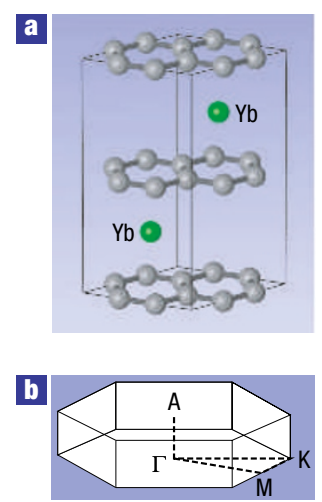
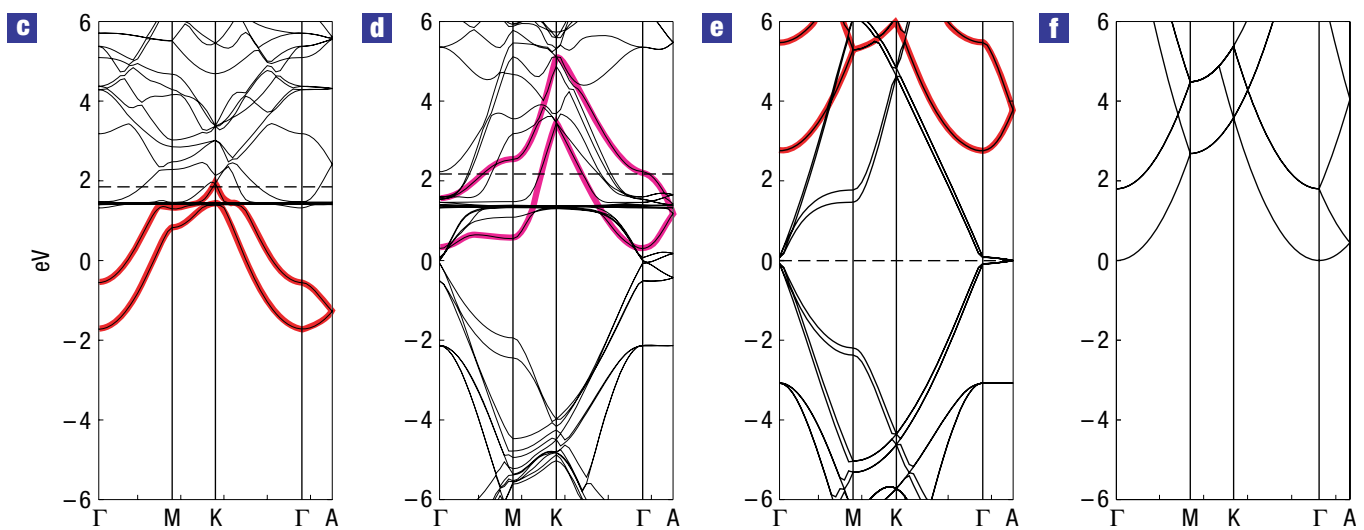

Figure 1 Band structure of $\mathrm{C}_{6} \mathrm{Yb}$ compared with various model systems as inferred from DFT-LDA. a, Atomic structure. $\mathbf{b}$, Geometry of the Brillouin zone of $\mathrm{C}_{6} \mathrm{Yb}$. c-f, Band structures of: $\mathbf{c}$, Expanded $\mathrm{Yb}$, with the lattice parameters and atomic positions corresponding to those in $\mathrm{C}_{6} \mathrm{Yb}$, so that the nearest $\mathrm{Yb}-\mathrm{Yb}$ distance is increased by $24 \%$ as compared with the pure face-centred-cubic structure; $\mathbf{d}$, the $\mathrm{C}_{6} \mathrm{Yb}$ compound; $\mathbf{e}$, the 'empty' graphite system, where the $\mathrm{Yb}$ atoms have been removed but the lattice constants are kept fixed; $\mathbf{f}$, free-electron bands, folded into the unit cell of $\mathrm{C}_{6} \mathrm{Yb}$. The free-electron-like band is marked with a thick red line, whereas pink lines indicate hybridized bands with significant charge density in the interlayer region. The band structures (except for the free-electron bands) are aligned with respect to the core levels and in each case the Fermi level is shown with a dashed line. Including correlation effects for the localized $4 f$ orbitals in the LDA+U method results only in the expected downward rigid shift of the energy of the filled $4 f$ levels (not shown).
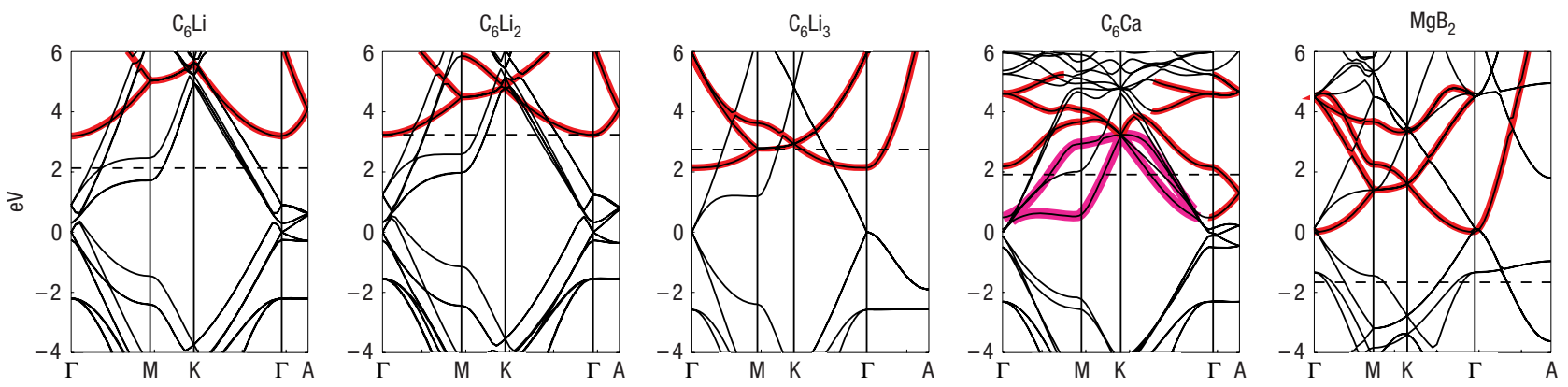

Figure 2 Band structures of the Li series of GICs, $\mathbf{C}_{6} \mathbf{C a}$ and $\mathbf{M g B}_{2} . \mathrm{MgB}_{2}$ is shown in a supercell that matches the others in the plane of the graphene sheets. The interlayer band is marked with a thick red line, whereas pink lines indicate hybridized bands with significant charge density in the interlayer region. The band structures are aligned by the crossing of the $\pi$ bands, in each case the Fermi level is shown with a dashed line.

in its occupancy in the $\mathrm{C}_{2} \mathrm{Li}$ system. Indeed, combining the available data in Table 1, the coincidence of superconductivity in the GICs with the occupation of the interlayer state is striking.

Studies of the free-electron-like interlayer state in pure graphite and GICs have a long history. Although present in early bandstructure calculations in the pure system, the significance of the (unoccupied) band in graphite and $\mathrm{C}_{6} \mathrm{Li}$ was first emphasized in refs 11,12 and, subsequently, its existence was confirmed experimentally in both graphite ${ }^{13}$ and $\mathrm{C}_{6} \mathrm{Li}$ (ref. 14). (The same band has been equivalently referred to as a metallic ' $s d$ band' in ref. 15.) Referring to the Li-based GICs, it is apparent that the energy and, therefore, also the occupancy of this band can vary considerably with respect to the other bands present in the above systems.

It is notable that the dispersive $5 d$ band of $\mathrm{Yb}$ in the expanded metal (Fig. 1c) and the interlayer state in pure graphite (Fig. 1e) both bear strong resemblance to the dispersion of the band of free electrons in the absence of external potential (Fig. 1f). Indeed, we can understand the important features of this nearly freeelectron-like state by studying the 'empty' graphite system with the intercalant ions removed. In Fig. 4 we show the effect of charging and layer separation on the energy of the resulting interlayer band. An increase in the $c$-axis lattice constant and in the accumulated charge both lead to a lowering of the band energy and, thus, an increase in its occupancy. It is interesting to note that, for a wide range of experimentally known GICs, the interlayer band energy follows closely that of the pure graphite model.

Motivated by preliminary electronic structure calculations, theoretical studies ${ }^{16,17}$ have emphasized the significance of partially occupied two-dimensional graphite $\pi^{*}$ bands and threedimensional interlayer bands for superconductivity. Indeed, these studies have shown that aspects of superconductivity in some GICs can be described well by a two-band phenomenology. Yet the underlying microscopic pairing mechanism remains in question. Significantly, unlike the fullerene-based superconducting compounds, the $\pi^{*}$ bands are decoupled from the out-of-plane lattice vibrations of the graphene sheets and couple only weakly to the in-plane modes. By contrast, one may expect the interlayer band to engage with lattice vibrations of the metal ions and, through their hybridization with the $\pi^{*}$ bands, the graphene sheets. Indeed, numerical calculations have shown the presence of strong electron-phonon coupling in $\mathrm{C}_{6} \mathrm{Ca}$, facilitated by the occupation 

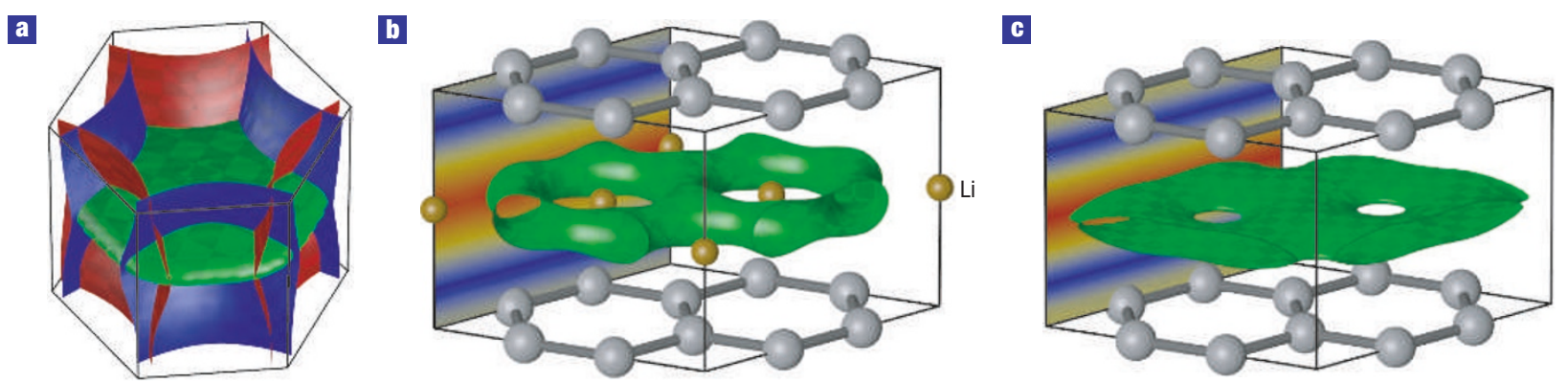

Figure 3 Structure of the interlayer state. a, Fermi surface of the $C_{2}$ Li system as inferred from the band-structure calculation. In its native state, the Fermi surface of a single graphene sheet collapses onto two points in the Brillouin zone. Electron-doped, the Fermi surface expands to form a set of two cylindrical electron-like surfaces associated with the $\pi^{*}$ bands that enclose the singular points in the spectrum (red and blue). By contrast, the interlayer band, which adopts a nearly free-electron-like structure parallel to the planes near the $\Gamma$ point, acquires a more complex extended character at the Fermi surface (green). $\mathbf{b}$, An isosurface of the charge density associated with the lowest interlayer band in $\mathrm{C}_{2} \mathrm{Li}$ at the $\Gamma$ point. The colourmap on the left plane shows the projection of the band density, with blue corresponding to low and red to high electron density. The spatial structure of the interlayer band is essentially identical to that of its unoccupied analogue in pure graphite, which is shown in $\mathbf{c}$.

Table 1 Table recording the coincidence of the interlayer state occupation in GICs and the phenomenon of superconductivity as inferred from the results of band-structure calculation and experiment. According to the electronic structure calculations and the observed phenomenology, one would predict superconductivity in the GIC $\mathrm{C}_{6} \mathrm{Ba}$ (refs 28,29).

\begin{tabular}{lclcc}
\hline GIC & $\begin{array}{c}\text { Electron } \\
\text { doping }\end{array}$ & $\begin{array}{l}\text { Interlayer } \\
\text { separation }(\AA)\end{array}$ & $\begin{array}{c}\text { Interlayer } \\
\text { band occupation }\end{array}$ & $\begin{array}{c}T_{\mathrm{c}} \\
(\mathrm{K})\end{array}$ \\
\hline Graphite & 0 & 3.35 & No & - \\
$\mathrm{C}_{6} \mathrm{Li}$ & $1 / 6$ & 3.7 & No & - \\
$\mathrm{C}_{3} \mathrm{Li}$ & $1 / 3$ & 3.7 & No & - \\
$\mathrm{C}_{2} \mathrm{Li}$ & $1 / 2$ & 3.7 & Yes & 1.9 \\
$\mathrm{C}_{16} \mathrm{~K}$ & $1 / 16$ & 5.2 & No & - \\
$\mathrm{C}_{8} \mathrm{~K}$ & $1 / 8$ & 5.2 & Yes & 0.14 \\
$\mathrm{C}_{6} \mathrm{Ca}$ & $1 / 3$ & 4.6 & Yes & 11.5 \\
$\mathrm{C}_{6} \mathrm{Yb}$ & $1 / 3$ & 4.7 & Yes & 6.5 \\
$\mathrm{C}_{6} \mathrm{Ba}$ & $1 / 3$ & 5.25 & Yes & $?$ \\
\hline
\end{tabular}

of the free-electron-like band ${ }^{8}$. Whether an electron-phonon mechanism alone can explain the broad distribution of $T_{\mathrm{c}}$ observed across the range of GICs, as well as the staging dependence ${ }^{18}$, remains a question for future investigation. In this context, it is interesting to note that, in those compounds with a low transition temperature, the interlayer band remains nearly orthogonal to the $\pi^{*}$ bands, as it is in pure graphite, whereas in $\mathrm{C}_{6} \mathrm{Yb}$ and $\mathrm{C}_{6} \mathrm{Ca}$ the hybridization is strong.

Intriguingly, through its weak coupling with the graphene layer, one may expect occupancy of the intrinsic interlayer band of graphite to provide an ideal environment for soft charge fluctuations that could promote ( $s$-wave) superconductivity by means of an excitonic pairing mechanism ${ }^{19}$. Indeed, the same weak coupling diminishes the potential for charge density wave instability, which might otherwise compete with the superconducting pair correlations. In this context, it is interesting to note that a parallel pairing mechanism involving the exchange of acoustic plasmons has been suggested ${ }^{20}$ and was much discussed after the discovery of superconductivity in the cuprates. The model has been re-evaluated for layered metals ${ }^{21}$. Bearing in mind that low-frequency plasma oscillations will, of course, couple to the ionic positions-conventional acoustic phonons can be viewed as simply coupled ionic and electronic plasma oscillations-such processes present an effective mechanism to enhance electronphonon coupling.

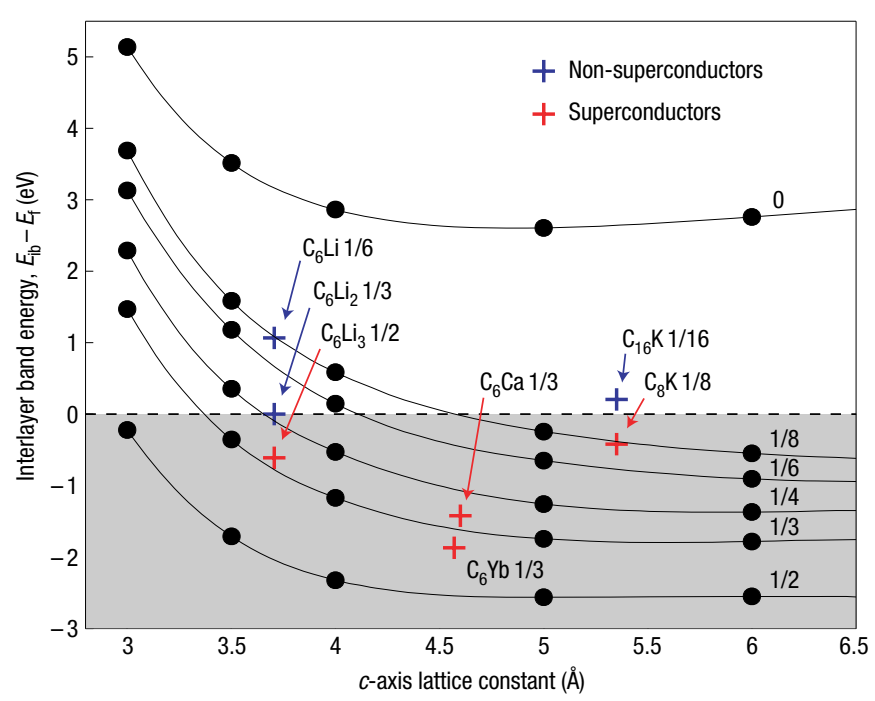

Figure 4 The dependence of the interlayer band energy (at the $\Gamma$ point) of the empty graphite system with changing $c$-axis spacing for different electron dopings. The calculation of the electron-doped system using periodic boundary conditions requires overall charge neutrality, enforced by a uniform positive background charge. Data points corresponding to different GICs are superposed on the figure, red and blue crosses indicate whether the compound is found to be superconducting or not, respectively. The fractions show the electron doping of the graphite sheets in units of electrons per carbon atom. The interlayer band is occupied in the systems that fall into the shaded area.

Recognizing the auspicious role played by the free-electronlike state in the superconducting GICs, it is tempting to look for a common phenomenology in the isoelectronic family of boron intercalates and, in particular, the well-studied superconducting compound $\mathrm{MgB}_{2}$, which exhibits a $T_{\mathrm{c}}=39 \mathrm{~K}$ (ref. 22). Although the material indeed possesses interlayer states, the band structure (Fig. 2) shows that it remains unoccupied. However, in contrast to $\mathrm{C}_{6} \mathrm{Yb}$ and the other superconducting GICs, in this case charge carriers are transferred from the $\sigma$ to the $\pi^{*}$ band. The resulting band of hole-like $\sigma$ states couples strongly to the host lattice providing a natural environment to realize a high-temperature phonon-mediated superconductivity. 
Finally, it is interesting to note that the interlayer states find their analogue as nearly free-electron-like states in the carbon nanotube system: early theoretical ${ }^{23}$ and experimental ${ }^{24}$ investigations of the alkali-metal nanotube intercalates indicated a phenomenology in doping dependence similar to the GICs. On this background, an investigation of possible superconductivity in electron-doped carbon nanotubes would seem to be a timely and worthwhile enterprise.

\section{METHODS}

The results reported here were obtained from an implementation of the LDA in the framework of the CASTEP package $e^{25}$. The methodology, which includes the use of ultrasoft pseudopotentials, has been validated in many environments including the rare-earth compounds ${ }^{26}$. Here, we take advantage of the fact that, in using ultrasoft pseudopotentials, one can exercise a considerable degree of freedom in deciding which electron shells to treat as fixed core and which to treat as valence, free to participate in bonding.

The numerical results presented for $\mathrm{C}_{6} \mathrm{Yb}$ were computed by treating all of the electrons below the $5 s$ level for $\mathrm{Yb}$ and below the $2 s$ level for $\mathrm{C}$ as core. Plane waves up to $480 \mathrm{eV}$ were used to expand the electronic states and the Brillouin zone was sampled with a $12 \times 12 \times 6$ mesh. (A test calculation using a $600 \mathrm{eV}$ cutoff did not yield appreciably different results.) To check for possible correlation effects, we used the LDA+U method as implemented in the PWSCF package ${ }^{27}$ with the parameter $\mathrm{U}$ varying between 3 and $7 \mathrm{eV}$.

Received 18 March 2005; accepted 26 August 2005; published 29 September 2005.

\section{References}

1. Dresselhaus, M. S. \& Dresselhaus, G. Intercalation compounds of graphite. Adv. Phys. 51, $1-186$ (2002).

2. Koike, Y., Suemetsu, H., Higuchi, K. \& Tanuma, S. Superconductivity in graphite-alkali metal intercalation compounds. Physica B+C 99, 503-508 (1980).

3. Hannay, N. B. et al. Superconductivity in graphite compounds. Phys. Rev. Lett. 14, 225-226 (1965)

4. Belash, I. T., Bronnikov, A. D., Zharikov, O. V. \& Palnichenko, A. V. On the superconductivity of graphite intercalation compounds with sodium. Solid State Commun. 64, 1445-1447 (1987).

5. Weller, T., Ellerby, M., Saxena, S. S., Smith, R. \& Skipper, N. Superconductivity in the intercalated graphite compounds $\mathrm{C}_{6} \mathrm{Yb}$ and $\mathrm{C}_{6} \mathrm{Ca}$. Nature Phys. 1, 39-41 (2005).

6. Payne, M. C., Teter, M. P., Allan, D. C., Arias, T. A. \& Joannopoulos, J. D. Iterative minimization techniques for ab initio total-energy calculations: molecular dynamics and conjugate gradients. Rev. Mod. Phys. 64, 1045-1097 (1992).

7. Perdew, J. \& Zunger, A. Self-interaction correction to density-functional approximations for many-electron systems. Phys. Rev. B 23, 5048-5079 (1981).

8. Calandra, M. \& Mauri, F. Superconducitivity in $\mathrm{C}_{6}$ Ca. Preprint at http://arxiv.org/abs/cond-mat/0506082 (2005)
9. Koma, A., Miki, K., Suematsu, H., Ohno, T. \& Kamimura, H. Density-of-states investigation of $\mathrm{C}_{8} \mathrm{~K}$ and occurrence of the interlayer band. Phys. Rev. B 34, 2434-2438 (1986).

10. Belash, I. T., Bronnikov, A. D., Zharikov, O. V. \& Pal'nichenko, A. V. Superconductivity of graphite intercalation compound with lithium $\mathrm{C}_{2}$ Li. Solid State Commun. 69, 921-923 (1989).

11. Posternak, M., Baldereschi, A., Freeman, A. J., Wimmer, E. \& Weinert, M. Prediction of electronic interlayer states in graphite and reinterpretation of alkali bands in graphite intercalation compounds. Phys. Rev. Lett. 50, 761-764 (1983).

12. Holzwarth, N. A. W., Louie, S. G. \& Rabii, S. Interlayer states in graphite and in alkali-metal-graphite intercalation compounds. Phys. Rev. B 30, 2219-2222 (1984).

13. Reihl, B., Gimzewski, J. K., Nicholls, J. M. \& Tosatti, E. Unoccupied electronic states of graphite as probed by inverse-photoemission and tunneling spectroscopy. Phys. Rev. B 33, 5770-5773 (1986)

14. Fauster, Th., Himpsel, F. J., Fischer, J. E. \& Plummer, E. W. Three-dimensional energy band in graphite and lithium-intercalated graphite. Phys. Rev. Lett. 51, 430-433 (1983).

15. Molodtsov, S. L., Laubschat, C., Richter, M., Gantz, Th. \& Shikin, A. M. Electronic structure of Eu and $\mathrm{Yb}$ graphite intercalation compounds. Phys. Rev. B 53, 16621-16630 (1996).

16. Al Jishi, R. Model for superconductivity in graphite intercalation compounds. Phys. Rev. B 28, $112-116$ (1983).

17. Jishi, R. A. \& Dresselhaus, M. S. Superconductivity in graphite intercalation compounds. Phys. Rev. B 45, 12465-12469 (1992).

18. Iye, Y. \& Tanuma, S. Superconductivity of graphite intercalation compounds with alkali-metal amalgams. Phys. Rev. B 25, 4583-4592 (1982).

19. Allender, D., Bray, J. \& Bardeen, J. Model for an exciton mechanism of superconductivity. Phys. Rev. B 7, 1020-1029 (1973).

20. Takada, Y. Plasmon mechanism of superconductivity in two- and three-dimensional electron systems. J. Phys. Soc. Jpn 45, 786-794 (1978).

21. Bill, A., Morawitz, H. \& Kresin, V. Z. Electronic collective modes and superconductivity in layered conductors. Phys. Rev. B 68, 144519 (2003).

22. Nagamatsu, J., Nakagawa, N., Muranaka, T., Zenitani, Y. \& Akimitsu, J. Superconductivity at $39 \mathrm{~K}$ in magnesium diboride. Nature 410, 63-64 (2001).

23. Miyamoto, Y., Rubio, A., Blase, X., Cohen, M. L. \& Louie, S. G. Ionic cohesion and electron doping of thin carbon tubules with alkali atoms. Phys. Rev. Lett. 74, $2993-2996$ (1995).

24. Shimoda, H. et al. Lithium intercalation into opened single-wall carbon nanotubes: Storage capacity and electronic properties. Phys. Rev. Lett. 88, 015502 (2002).

25. Segall, M. D. et al. First-principles simulation: ideas, illustrations and the CASTEP code. J. Phys. C. 14, 2717-2744 (2002).

26. Pickard, C. J. et al. Structural properties of lanthanide and actinide compounds within the plane wave pseudopotential approach. Phys. Rev. Lett. 85, 5122-5125 (2000).

27. Baroni, S., Dal Corso, A., de Gironcoli, S. \& Giannozzi, P. http://www.pwscf.org.

28. Hérold, A. in Intercalated Layered Materials Vol. 6 (ed. Lévy, F.) 323-421 (Reidel, Dordrecht, 1979).

29. Guérard, D. \& Hérold, A. Synthèse directe de composés d'insertion du baryum dans le graphite. C.R. Acad. Sci. Paris, Ser. C 279, 455-456 (1974).

\section{Acknowledgements}

We are grateful to S. S. Saxena and M. Ellerby for bringing the $\mathrm{C}_{6} \mathrm{Yb}$ and $\mathrm{C}_{6} \mathrm{Ca}$ compounds to our attention and to G. G. Lonzarich for contributing to our understanding.

Correspondence and requests for materials should be addressed to G.C.

\section{Competing financial interests}

The authors declare competing financial interests: details accompany the paper on www.nature.com/naturephysics.

Reprints and permission information is available online at http://npg.nature.com/reprintsandpermissions/ 